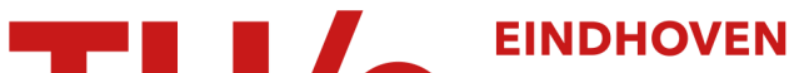 UNIVERSITY OF TECHNOLOGY
}

\section{Sound propagation in slowly varying 2D duct with shear flow}

Citation for published version (APA):

Rienstra, S. W. (2016). Sound propagation in slowly varying 2D duct with shear flow. (CASA-report; Vol. 1613). Technische Universiteit Eindhoven.

Document status and date:

Published: 01/05/2016

\section{Document Version:}

Publisher's PDF, also known as Version of Record (includes final page, issue and volume numbers)

\section{Please check the document version of this publication:}

-A submitted manuscript is the version of the article upon submission and before peer-review. There can be important differences between the submitted version and the official published version of record. People interested in the research are advised to contact the author for the final version of the publication, or visit the $\mathrm{DOI}$ to the publisher's website.

- The final author version and the galley proof are versions of the publication after peer review.

- The final published version features the final layout of the paper including the volume, issue and page numbers.

Link to publication

\section{General rights}

Copyright and moral rights for the publications made accessible in the public portal are retained by the authors and/or other copyright owners and it is a condition of accessing publications that users recognise and abide by the legal requirements associated with these rights.

- Users may download and print one copy of any publication from the public portal for the purpose of private study or research.

- You may not further distribute the material or use it for any profit-making activity or commercial gain

- You may freely distribute the URL identifying the publication in the public portal.

If the publication is distributed under the terms of Article $25 \mathrm{fa}$ of the Dutch Copyright Act, indicated by the "Taverne" license above, please follow below link for the End User Agreement:

www.tue.nl/taverne

Take down policy

If you believe that this document breaches copyright please contact us at:

openaccess@tue.nl

providing details and we will investigate your claim. 


\section{EINDHOVEN UNIVERSITY OF TECHNOLOGY}

Department of Mathematics and Computer Science

CASA-Report 16-13

May 2016

Sound Propagation in Slowly Varying

2D Duct with Shear Flow

by

S.W. Rienstra

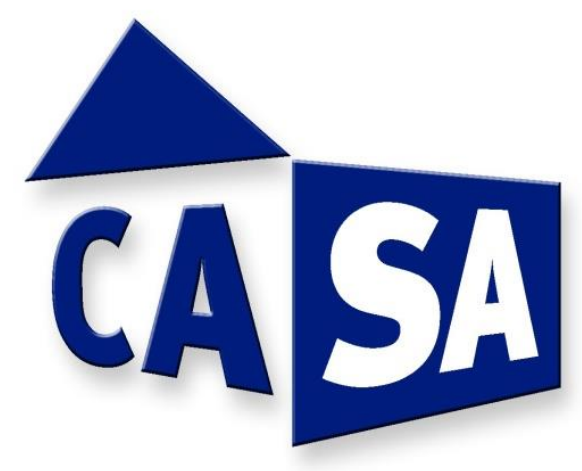

Centre for Analysis, Scientific computing and Applications

Department of Mathematics and Computer Science

Eindhoven University of Technology

P.O. Box 513

5600 MB Eindhoven, The Netherlands

ISSN: 0926-4507 

AIAA-2016-2925

22 $2^{\text {nd }}$ AIAA/CEAS Aeroacoustics Conference, 31 May - 01 June 2016, Lyon, France

\title{
Sound Propagation in Slowly Varying 2D Duct with Shear Flow
}

\author{
Sjoerd W. Rienstra*
}

\begin{abstract}
A slowly varying modes solution of WKB type is derived for the problem of sound propagation in a slowly varying $2 \mathrm{D}$ duct with linearly sheared mean flow. Both the slowly varying mean flow equations and the Pridmore-Brown equation for the modes can be solved analytically exactly.
\end{abstract}

\section{Introduction}

Cound propagation in uniformly lined straight ducts with uniform mean flow is well established $S$ by its analytically exact description in duct modes $[1,2,3]$. In cross-wise nonuniform flow there are still modes, although the (Pridmore-Brown) equation that describes them is in general not solvable in terms of standard functions $[4,5,6,7,8,9]$ and has to be solved numerically.

These modal solutions provide insight, but the important effects due to the variation of the duct geometry and the corresponding mean flow cannot be described exactly. The inherently smooth variation of a flow duct, however, provides a small parameter (the slenderness of the duct wall variation) that allows asymptotic solutions of WKB type in the form of slowly varying modes in potential mean flow (asymptotically equivalent to quasi 1D gas flow in slender ducts) $[10,11,12,13,14,15,16,17,19]$.

This approach have been favourably compared with fully numerical solutions [18], and has also otherwise been incorporated in full numerical approaches [17].

Much more difficult is it to combine slowly varying modes with non-uniform shear flow. The main problem is that a general theory for sheared flow in slowly varying ducts does not exist. An exception is the special case of uniform axial flow with solid body rotation, considered by Cooper and Peake in [20], but the mean flow still requires the numerical integration of a ordinary differential equation. If the duct varies just in impedance, rather than its geometry, like in [8], the problem of the mean flow is avoided and a rather general result is again possible. Another problem is that with nonuniform mean flow the acoustic equation (a form of the Pridmore-Brown equation) has in general no solutions in terms of standard functions, leading to a rather extended numerical component of the essentially analytical asymptotic approximation.

In the present paper we will try to explore a special case of non-uniform shear flow in a slowly varying hard-walled duct, where both problems do not exist. As was found by Goldstein and Rice [21], the acoustic equations for 2D linear shear flow and uniform density, pressure and soundspeed, can be solved exactly by Weber's Parabolic Cylinder functions, while the corresponding

\footnotetext{
*Associate Professor, Department of Mathematics \& Computer Science, Eindhoven University of Technology, Netherlands, Senior Member AIAA.

Copyright (C) 2016 by S.W. Rienstra. Published by the American Institute of Aeronautics and Astronautics, Inc. with permission.
} 
slowly varying mean flow is exactly solvable in a manner reminiscent of the quasi 1D gas dynamics solution.

\section{The problem}

\section{II.A. The equations}

In the acoustic realm of a perfect gas that we will consider, we have for pressure $\tilde{p}$, velocity $\tilde{\boldsymbol{v}}$, density $\tilde{\rho}$, entropy $\tilde{s}$, and soundspeed $\tilde{c}$

$$
\begin{gathered}
\frac{\mathrm{d} \tilde{\rho}}{\mathrm{d} t}=-\tilde{\rho} \nabla \cdot \tilde{\boldsymbol{v}}, \quad \tilde{\rho} \frac{\mathrm{d} \tilde{\boldsymbol{v}}}{\mathrm{d} t}=-\nabla \tilde{p}, \quad \frac{\mathrm{d} \tilde{s}}{\mathrm{~d} t}=0, \\
\tilde{s}=C_{V} \log \tilde{p}-C_{P} \log \tilde{\rho}, \quad \tilde{c}^{2}=\frac{\gamma \tilde{p}}{\tilde{\rho}}, \quad \gamma=\frac{C_{P}}{C_{V}} .
\end{gathered}
$$

where $\gamma, C_{P}$ and $C_{V}$ are gas constants. $C_{V}$ is the heat capacity at constant volume, $C_{P}$ is the heat capacity at constant pressure, and $\gamma=C_{P} / C_{V}$. When we have a stationary mean flow with unsteady time-harmonic perturbations of frequency $\omega$, given, in the usual complex notation, by

$$
\tilde{\boldsymbol{v}}=\boldsymbol{V}+\operatorname{Re}\left(\boldsymbol{v} \mathrm{e}^{\mathrm{i} \omega t}\right), \tilde{p}=P+\operatorname{Re}\left(p \mathrm{e}^{\mathrm{i} \omega t}\right), \tilde{\rho}=D+\operatorname{Re}\left(\rho \mathrm{e}^{\mathrm{i} \omega t}\right), \tilde{s}=S+\operatorname{Re}\left(s \mathrm{e}^{\mathrm{i} \omega t}\right),
$$

$(\omega>0)$ and linearise for small amplitude, we obtain for the mean flow

$$
\begin{gathered}
\nabla \cdot(D \boldsymbol{V})=0, \quad D(\boldsymbol{V} \cdot \nabla) \boldsymbol{V}=-\nabla P, \\
(\boldsymbol{V} \cdot \nabla) S=0, \quad S=C_{V} \log P-C_{P} \log D, \quad C^{2}=\frac{\gamma P}{D},
\end{gathered}
$$

and the perturbations

$$
\begin{gathered}
\mathrm{i} \omega \rho+\nabla \cdot(\boldsymbol{V} \rho+\boldsymbol{v} D)=0, \\
D(\mathrm{i} \omega+\boldsymbol{V} \cdot \nabla) \boldsymbol{v}+D(\boldsymbol{v} \cdot \nabla) \boldsymbol{V}+\rho(\boldsymbol{V} \cdot \nabla) \boldsymbol{V}=-\nabla p, \\
(\mathrm{i} \omega+\boldsymbol{V} \cdot \nabla) s+\boldsymbol{v} \cdot \nabla S=0, \\
s=\frac{C_{V}}{P} p-\frac{C_{P}}{D} \rho=\frac{C_{V}}{P}\left(p-C^{2} \rho\right) .
\end{gathered}
$$

\section{II.B. Nondimensionalisation}

Without further change of notation, we will assume throughout this paper that the problem is made dimensionless: lengths on a typical duct height $a_{\infty}$, velocities on a typical sound speed $c_{\infty}$, time on $a_{\infty} / c_{\infty}$, densities on a typical density $\rho_{\infty}$, pressures on $\rho_{\infty} c_{\infty}^{2}$ and entropy on $C_{V}$. Note that the corresponding actual pressure $p_{\infty}$ is given by $p_{\infty}=\rho_{\infty} c_{\infty}^{2} / \gamma$.

\section{II.C. The geometry}

The domain of interest is a $2 \mathrm{D}$ duct $\mathcal{V}$ of slowly varying cross section $g(X) \leqslant y \leqslant h(X)$, where $0<h-g=\mathcal{O}(1) . X=\varepsilon x$ is a so-called slow variable while $\varepsilon$ is small. At the duct top surface $y=h$ the gradient

$$
\nabla(y-h(X))=\boldsymbol{e}_{y}-\varepsilon \boldsymbol{e}_{x} h_{X}
$$

$$
2 \text { of } 17
$$




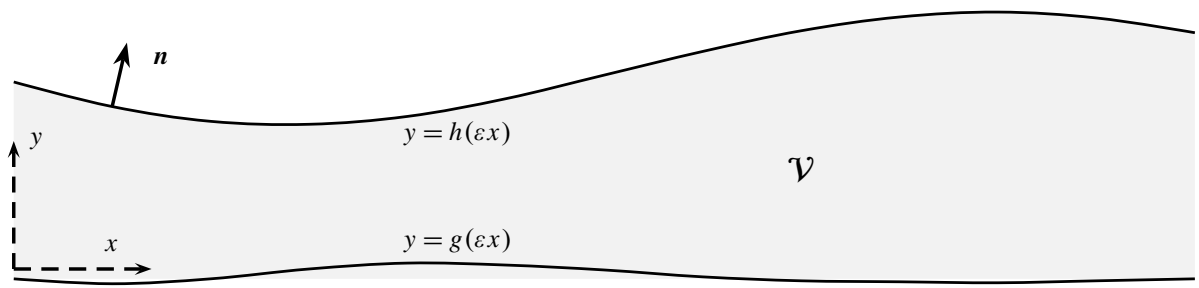

Figure 1. Sketch of geometry: 2-dimensional slowly varying duct

(where an index denotes a partial derivative) is a vector normal to the surface, so the outward normal of the top surface is given by

$$
\boldsymbol{n}=\frac{\boldsymbol{e}_{y}-\varepsilon \boldsymbol{e}_{x} h_{X}}{\sqrt{1+\varepsilon^{2} h_{X}^{2}}}=\boldsymbol{e}_{y}-\varepsilon \boldsymbol{e}_{x} h_{X}+\mathcal{O}\left(\varepsilon^{2}\right) .
$$

A similar formula applies to the bottom surface $y=g$.

\section{II.D. Boundary conditions}

The duct wall is impermeable to the mean flow, so we have the mean flow boundary conditions

$$
V-\varepsilon g_{X} U=0 \text { at } y=g(X), \text { and } V-\varepsilon h_{X} U=0 \text { at } y=h(X) .
$$

If we denote the mean flow by $\boldsymbol{V}=U \boldsymbol{e}_{x}+V \boldsymbol{e}_{y}$, with the axial component $U$ and the cross-wise component $V$, the mean flow mass flux, given by

$$
\int_{g}^{h} D U \mathrm{~d} y=\mathcal{F},
$$

is independent of $x$, where $\mathcal{F}$ is (after non-dimensionalisation) a constant $\mathcal{O}(1)$. The mean flow is assumed to be determined by the slowly varying geometry only.

The acoustic boundary condition of the hard wall is similar to the mean flow and given by

$$
\boldsymbol{v} \cdot \boldsymbol{n}=0,
$$

which amounts to

$$
v-\varepsilon g_{X} u=0 \text { at } y=g(X), \text { and } v-\varepsilon h_{X} u=0 \text { at } y=h(X) .
$$

\section{Mean flow}

For a potential mean flow the above conditions would be sufficient to define the flow [15]. In contrast, a sheared (vortical) mean flow, like we have here, is not defined yet without more conditions. The type of velocity profile we will consider is an almost linear shear flow, like $U_{y} \propto$ a coefficient that vary with $X$, to obey the condition of fixed mass flux and varying wall normal. So we postulate a mean flow that is to leading order given by

$$
U(X, y)=\tau(X)+\sigma(X)(y-g(X)),
$$

$$
3 \text { of } 17
$$


where $\sigma$ and $\tau$ are to be determined.

We do not assume a general temperature (sound speed, density) profile, but a homentropic flow with $S$ (the same) constant, such that

$$
\gamma P=D^{\gamma}, \quad D C^{2}=\gamma P, \quad C^{2}=D^{\gamma-1} .
$$

Since we assumed the mean flow to be determined by the slowly varying geometry only, we write all flow variables as a function of $(X, y ; \varepsilon)$, and expand each in a regular Poincaré expansion in powers of $\varepsilon^{2}$ (the small parameter that appears in the equations). From elementary order of magnitude considerations it follows that $U=\mathcal{O}(1), V=\mathcal{O}(\varepsilon), D=\mathcal{O}(1), C=\mathcal{O}(1)$, and $P=\mathcal{O}(1)$. So we have

$$
\begin{gathered}
U=U_{0}+\mathcal{O}\left(\varepsilon^{2}\right), \quad V=\varepsilon V_{0}+\mathcal{O}\left(\varepsilon^{3}\right), \\
D=D_{0}+\mathcal{O}\left(\varepsilon^{2}\right), \quad P=P_{0}+\mathcal{O}\left(\varepsilon^{2}\right), \quad C=C_{0}+\mathcal{O}\left(\varepsilon^{2}\right),
\end{gathered}
$$

where each term in the expansion is independent of $\varepsilon$. For notational convenience we leave out the 0 subscript, because higher orders will not be considered.

We substitute these expansions in the conservation equations and collect the terms of like powers of $\varepsilon$. Then we get to leading order

$$
\begin{aligned}
(D U)_{X}+(D V)_{y} & =0, \\
D\left(U U_{X}+V U_{y}\right)+P_{X} & =0, \\
P_{y} & =0,
\end{aligned}
$$

with boundary conditions

$$
V-g_{X} U=0 \text { at } y=g \text { and } V-h_{X} U=0 \text { at } y=h(X) .
$$

From $P=P(X)$ is also $D=D(X)$ and $C=C(X)$, and so $P_{X}=C^{2} D_{X}=D^{\gamma-1} D_{X}$. Therefore

$$
\begin{aligned}
(D U)_{X}+D V_{y} & =0 \\
D U U_{X}+D V U_{y}+D^{\gamma-1} D_{X} & =0 .
\end{aligned}
$$

This leads to

$$
D V=g_{X} D U(g)-\int_{g}^{y}(D U)_{X} \mathrm{~d} y^{\prime},
$$

which by construction satisfies the boundary condition at $y=g$, and satisfies indeed at $y=h$

$$
D V-D U h_{X}=-\frac{\mathrm{d}}{\mathrm{d} X} \int_{g}^{h} D U \mathrm{~d} y=-\frac{\mathrm{d}}{\mathrm{d} X} \mathcal{F}=0 .
$$

Now substitute

$$
U=\tau+\sigma(y-g)
$$

then

$$
D V=D g_{X} U-(D \tau)_{X}(y-g)-\frac{1}{2}(D \sigma)_{X}(y-g)^{2}
$$

$$
4 \text { of } 17
$$


and

$$
D \tau \tau_{X}+D^{\gamma-1} D_{X}+\left(D \sigma_{X}-D_{X} \sigma\right)\left(\tau(y-g)+\frac{1}{2} \sigma(y-g)^{2}\right)=0 .
$$

This requires (at $y=g$ ) that

$$
D \tau \tau_{X}+D^{\gamma-1} D_{X}=0
$$

and therefore

$$
D \sigma_{X}-D_{X} \sigma=0 .
$$

This is satisfied if for any (problem dependent) constants $\lambda$ and $E$

$$
\sigma(X)=\lambda D(X)
$$

and the Bernoulli-like ${ }^{1}$ equation

$$
\frac{1}{2} \tau^{2}+\frac{1}{\gamma-1} D^{\gamma-1}=E
$$

Eliminate $\tau$ from

$$
\int_{g}^{h} D U \mathrm{~d} y=D \int_{g}^{h} U \mathrm{~d} y=D(h-g)\left(\tau+\frac{1}{2} \sigma(h-g)\right)=\mathcal{F}
$$

to get

$$
\tau=\frac{\mathcal{F}}{D(h-g)}-\frac{1}{2} \sigma(h-g) .
$$

For given geometry $h$ and $g$ and mean flow constants $E, \lambda$ and $\mathcal{F}$ we have then

$$
\frac{1}{2}\left(\frac{\mathcal{F}}{D(h-g)}-\frac{1}{2} \lambda D(h-g)\right)^{2}+\frac{1}{\gamma-1} D^{\gamma-1}=E,
$$

to be solved per $X$ for $D$. ( $E$ and $\mathcal{F}$ have to be chosen carefully to avoid supersonic parts of the flow.) Then we have $\tau, \sigma, U$ and $C$ like given above, and the derivatives

$$
D_{X}=\frac{D \tau U(h)}{C^{2}-\tau U(h)} \cdot \frac{h_{X}-g_{X}}{h-g}, \quad \tau_{X}=-\frac{C^{2} D_{X}}{\tau D}, \quad \sigma_{X}=\lambda D_{X}, \quad U_{X}=\tau_{X}+\sigma_{X} y,
$$

from which $V$ and $V_{y}$ can be found like

$$
\begin{aligned}
& V=g_{X} U(y)+U(h) \frac{C^{2}-\tau U(y)}{C^{2}-\tau U(h)} \cdot \frac{h_{X}-g_{X}}{h-g}(y-g), \\
& V_{y}=g_{X} \sigma+U(h) \frac{C^{2}+\tau^{2}-2 \tau U(y)}{C^{2}-\tau U(h)} \cdot \frac{h_{X}-g_{X}}{h-g} .
\end{aligned}
$$

Note that there is apparently no slowly varying mean flow of linear shear with $\tau(X) \equiv 0$.

\footnotetext{
${ }^{1}$ Along the streamline $y=g$ Bernoulli's law applies.
} 


\section{Acoustic field}

\section{IV.A. Slowly varying modes}

With the mean flow variables expanded to first order, we have the acoustic equations

$$
\begin{aligned}
\mathrm{i} \omega \rho+U \rho_{x}+D\left(u_{x}+v_{y}\right) & =-\varepsilon\left[\rho\left(U_{X}+V_{y}\right)+u D_{X}+V \rho_{y}\right], \\
D\left(\mathrm{i} \omega u+U u_{x}\right)+D v \sigma+p_{x} & =-\varepsilon\left[-D^{-1} C^{2} D_{X} \rho+D\left(U_{X} u+V u_{y}\right)\right], \\
D\left(\mathrm{i} \omega v+U v_{x}\right)+p_{y} & =-\varepsilon\left[D V_{y} v+D V v_{y}\right], \\
\mathrm{i} \omega s+U s_{x} & =-\varepsilon V s_{y},
\end{aligned}
$$

where

$$
s=\frac{p}{P}-\frac{\gamma \rho}{D}=\frac{1}{P}\left(p-C^{2} \rho\right) .
$$

The hard wall boundary conditions at $y=g(\varepsilon x)$ and $y=h(\varepsilon x)$ are

$$
v(x, g)-\varepsilon g_{X} u(x, g)=0, \quad v(x, h)-\varepsilon h_{X} u(x, h)=0 .
$$

The assumption of a multiple scales solution is here equivalent to the WKB-Ansatz:

$$
\begin{aligned}
u & =A(X, y ; \varepsilon) \mathrm{e}^{-\mathrm{i} \int^{x} \mu(\varepsilon \xi ; \varepsilon) \mathrm{d} \xi}, \\
u_{x} & =\left(-\mathrm{i} \mu A+\varepsilon A_{X}\right) \mathrm{e}^{-\mathrm{i} \int^{x} \mu(\varepsilon \xi ; \varepsilon) \mathrm{d} \xi}, \\
v & =B(X, y ; \varepsilon) \mathrm{e}^{-\mathrm{i} \int^{x} \mu(\varepsilon \xi ; \varepsilon) \mathrm{d} \xi}, \\
p & =M(X, y ; \varepsilon) \mathrm{e}^{-\mathrm{i} \int^{x} \mu(\varepsilon \xi ; \varepsilon) \mathrm{d} \xi}, \\
\rho & =N(X, y ; \varepsilon) \mathrm{e}^{-\mathrm{i} \int^{x} \mu(\varepsilon \xi ; \varepsilon) \mathrm{d} \xi}, \\
s & =T(X, y ; \varepsilon) \mathrm{e}^{-\mathrm{i} \int^{x} \mu(\varepsilon \xi ; \varepsilon) \mathrm{d} \xi} .
\end{aligned}
$$

Introduce

$$
\Omega=\omega-\mu U,
$$

and substitute to obtain after some simplifications to $\mathcal{O}\left(\varepsilon^{2}\right)$

$$
\begin{aligned}
\mathrm{i} \Omega N+D\left(-\mathrm{i} \mu A+B_{y}\right) & =-\varepsilon\left[(U N+D A)_{X}+(V N)_{y}\right], \\
\mathrm{i} \Omega D A+D B \sigma-\mathrm{i} \mu M & =-\varepsilon D\left[\left(D^{-1} M+U A\right)_{X}+V A_{y}\right], \\
\mathrm{i} \Omega D B+M_{y} & =-\varepsilon D\left[U B_{X}+(V B)_{y}\right], \\
\mathrm{i} \Omega T & =-\varepsilon\left[U T_{X}+V T_{y}\right],
\end{aligned}
$$

with

$$
T=P^{-1}\left(M-C^{2} N\right)
$$

and boundary conditions

$$
B(X, g)-\varepsilon g_{X} A(X, g)=0, \quad B(X, h)-\varepsilon h_{X} A(X, h)=0 .
$$

We expand

$$
A=A_{0}+\varepsilon A_{1}+\ldots, B=B_{0}+\varepsilon B_{1}+\ldots, M=M_{0}+\varepsilon M_{1}+\ldots, N=N_{0}+\varepsilon N_{1}+\ldots,
$$


to obtain to leading order

$$
\begin{aligned}
\mathrm{i} \Omega N_{0}+D\left(-\mathrm{i} \mu A_{0}+B_{0, y}\right) & =0, \\
\mathrm{i} \Omega D A_{0}+D B_{0} \sigma-\mathrm{i} \mu M_{0} & =0, \\
\mathrm{i} \Omega D B_{0}+M_{0, y} & =0, \\
\mathrm{i} \Omega\left(M_{0}-C^{2} N_{0}\right) & =0,
\end{aligned}
$$

with boundary conditions

$$
B_{0}(X, g)=0, \quad B_{0}(X, h)=0 .
$$

This can be reduced to one equation in $M_{0}$ called the Pridmore-Brown equation:

$$
\Omega^{2}\left(\frac{M_{0, y}}{\Omega^{2}}\right)_{y}+\left(\frac{\Omega^{2}}{C^{2}}-\mu^{2}\right) M_{0}=0
$$

with boundary conditions

$$
M_{0, y}(X, g)=0, \quad M_{0, y}(X, h)=0 .
$$

Note that in equation (39) $X$ is a parameter, and we can write

$$
M_{0}(X, y)=Q(X) \psi(X, y) .
$$

This implies that $M_{0}$ is only determined up to a slowly varying amplitude. By normalising $\psi$ in some convenient way, we may uniquely define shape function $\psi$. However, the amplitude $Q$ is yet to be determined. We will derive an equation for it at the next order of expansion.

Before this, we will elaborate on the above equation (39), which can be solved in terms of standard functions.

\section{IV.B. Parabolic Cylinder Functions}

As was shown by Goldstein and Rice [21], this equation (39), due to the linear mean flow profile and provided $\sigma \neq 0$, can be solved in terms of Parabolic Cylinder functions (or Weber functions); see sections 19.16 and 19.17 of [23]. Introduce (in order to avoid square roots of imaginary quantities, we adopt a slightly different approach than [21]) the new variables

$$
\begin{aligned}
& \xi=\left(\frac{ \pm 2}{\mu \sigma C}\right)^{\frac{1}{2}} \Omega=\left(\frac{ \pm 2}{\mu \sigma C}\right)^{\frac{1}{2}}(\omega-\mu \tau-\mu \sigma(y-g)), \\
& \psi=\mathrm{e}^{\frac{1}{2} b \xi^{2}} \frac{\mathrm{d}}{\mathrm{d} \xi}\left[\mathrm{e}^{-\frac{1}{2} b \xi^{2}} \eta(\xi)\right]=\eta^{\prime}-b \xi \eta, \quad b=\frac{ \pm \mu C}{2 \sigma} .
\end{aligned}
$$

The \pm -sign is to be chosen conveniently. In the present case we will consider positive shear, i.e. $\sigma>0$, and propagating waves, i.e. only real $\mu$, and we will take $+\mu$ if $\mu$ is positive and $-\mu$ if $\mu$ is negative. In short, we take $\pm \mu=|\mu|$.

Now equation (39) becomes

$$
\frac{ \pm 2 \mu \sigma}{C} \mathrm{e}^{\frac{1}{2} b \xi^{2}} \xi^{2} \frac{\mathrm{d}}{\mathrm{d} \xi}\left[\frac{\mathrm{e}^{-\frac{1}{2} b \xi^{2}}}{\xi^{2}}\left(\eta^{\prime \prime}+\left(\frac{1}{4} \xi^{2}-b\right) \eta\right)\right]=0,
$$

which is evidently satisfied by solutions of

$$
\eta^{\prime \prime}+\left(\frac{1}{4} \xi^{2}-b\right) \eta=0
$$

7 of 17 
while

$$
\psi_{y}=\left(\frac{ \pm 2}{\mu \sigma C}\right)^{\frac{1}{2}} \mu \sigma\left(\frac{1}{4} \xi^{2} \eta+b \xi \eta^{\prime}\right) .
$$

This equation has an even and an odd solution

$$
\begin{aligned}
& \eta_{1}(\xi)=1+b \frac{\xi^{2}}{2 !}+\left(b^{2}-\frac{1}{2}\right) \frac{\xi^{4}}{4 !}+\left(b^{3}-\frac{7}{2} b\right) \frac{\xi^{6}}{6 !}+\ldots \\
& \eta_{2}(\xi)=\xi+b \frac{\xi^{3}}{3 !}+\left(b^{2}-\frac{3}{2}\right) \frac{\xi^{5}}{5 !}+\left(b^{3}-\frac{13}{2} b\right) \frac{\xi^{7}}{7 !}+\ldots
\end{aligned}
$$

and Wronskian $\eta_{1} \eta_{2}^{\prime}-\eta_{1}^{\prime} \eta_{2}=1$. The non-zero coefficients $a_{n}$ of $\xi^{n} / n$ ! are given by the recurrence relation

$$
a_{n+2}=b a_{n}-\frac{1}{4} n(n-1) a_{n-2},
$$

starting at $n=0$ for $a_{-2}=a_{-1}=0$, while $a_{0}=a_{1}=1$ yields the even coefficients for $\eta_{1}$ and the odd coefficients for $\eta_{2}$. More explicitly we have thus

$$
\begin{aligned}
& \eta_{1}(\xi)=\sum_{n=0}^{\infty} c_{n} \frac{\xi^{2 n}}{(2 n) !} \quad \text { where } \quad c_{n+1}=b c_{n}-n\left(n-\frac{1}{2}\right) c_{n-1}, \quad c_{-1}=0, c_{0}=1 \\
& \eta_{2}(\xi)=\sum_{n=0}^{\infty} d_{n} \frac{\xi^{2 n+1}}{(2 n+1) !} \quad \text { where } \quad d_{n+1}=b d_{n}-n\left(n+\frac{1}{2}\right) d_{n-1}, \quad d_{-1}=0, d_{0}=1
\end{aligned}
$$

Another standard form is by Whittaker's Parabolic function $D_{v}(x)$, which relates to $\eta_{1}$ and $\eta_{2}$ by

$$
\begin{aligned}
& \eta_{1}(\xi)=\frac{D_{\mathrm{i} b-\frac{1}{2}}\left(\mathrm{e}^{\frac{1}{4} \pi \mathrm{i}} \xi\right)+D_{\mathrm{i} b-\frac{1}{2}}\left(-\mathrm{e}^{\frac{1}{4} \pi \mathrm{i}} \xi\right)}{2 D_{\mathrm{i} b-\frac{1}{2}}(0)}, \\
& \eta_{2}(\xi)=\frac{D_{\mathrm{i} b-\frac{1}{2}}\left(\mathrm{e}^{\frac{1}{4} \pi \mathrm{i}} \xi\right)-D_{\mathrm{i} b-\frac{1}{2}}\left(-\mathrm{e}^{\left.\frac{1}{4} \pi \mathrm{i} \xi\right)}\right.}{-2 \mathrm{e}^{\frac{1}{4} \pi \mathrm{i}} D_{\mathrm{i} b+\frac{1}{2}}(0)} .
\end{aligned}
$$

For the time being, we will not use these forms.

With the sought $\eta(\xi)=c_{1} \eta_{1}(\xi)+c_{2} \eta_{2}(\xi)$, the equations to be solved for hard walls are

$$
\begin{aligned}
& \psi_{y}(X, g)=\left(\frac{ \pm 2}{\mu \sigma C}\right)^{\frac{1}{2}} \mu \sigma\left(\frac{1}{4} \xi_{g}^{2} \eta+b \xi_{g} \eta^{\prime}\right)=0 \text { at } \xi_{g}=\left(\frac{ \pm 2}{\mu \sigma C}\right)^{\frac{1}{2}}(\omega-\mu \tau), \\
& \psi_{y}(X, h)=\left(\frac{ \pm 2}{\mu \sigma C}\right)^{\frac{1}{2}} \mu \sigma\left(\frac{1}{4} \xi_{h}^{2} \eta+b \xi_{h} \eta^{\prime}\right)=0 \text { at } \xi_{h}=\left(\frac{ \pm 2}{\mu \sigma C}\right)^{\frac{1}{2}}(\omega-\mu \tau-\mu \sigma(h-g)),
\end{aligned}
$$

leading to the eigenvalue equation for $\mu$ (to be solved per $X$ )

$$
H_{1}\left(\xi_{g}\right) H_{2}\left(\xi_{h}\right)-H_{1}\left(\xi_{h}\right) H_{2}\left(\xi_{g}\right)=0,
$$

(assuming $\xi_{g} \xi_{h} \neq 0$ ) where

$$
H_{j}(\xi)=\frac{1}{4} \xi \eta_{j}(\xi)+b \eta_{j}^{\prime}(\xi) \text { and } c_{1}=H_{2}\left(\xi_{g}\right) c, c_{2}=-H_{1}\left(\xi_{g}\right) c .
$$

The equations for impedance walls are similar but evidently more involved. 
We note that the use of these Parabolic Cylinder Functions is numerically attractive only with a mean flow of high enough shear, i.e. $\sigma$ is not small. If $\sigma$ is small, the variables $\xi$ and $b$ become large, demanding asymptotic expansions and other special precautions in the numerical calculations of $\eta_{1}$ and $\eta_{2}$, and possibly making an analytically exact solution not always preferable over a direct numerical solution like in [9]. For $\sigma=0$, on the other hand, we have the exact solution with $U=\tau, \Omega=\omega-\mu \tau$,

$$
\psi_{y y}+\left(\frac{\Omega^{2}}{C^{2}}-\mu^{2}\right) \psi=0,
$$

and so, with the boundary conditions,

$$
\psi=\cos (\alpha y-\alpha g), \quad \alpha=\sqrt{\frac{\Omega^{2}}{C^{2}}-\mu^{2}}=\frac{n \pi}{h-g} \quad(n \in \mathbb{N}),
$$

and

$$
\mu=\frac{-\omega U \pm C \sqrt{\omega^{2}-\alpha^{2}\left(C^{2}-U^{2}\right)}}{C^{2}-U^{2}} .
$$

\section{IV.C. Final solution}

The next order is then

$$
\begin{aligned}
\mathrm{i} \Omega N_{1}+D\left(-\mathrm{i} \mu A_{1}+B_{1, y}\right) & =-\left[\left(U N_{0}\right)_{X}+\left(V N_{0}\right)_{y}+\left(D A_{0}\right)_{X}\right], \\
\mathrm{i} \Omega D A_{1}+D B_{1} \sigma-\mathrm{i} \mu M_{1} & =-\left[D\left(D^{-1} M_{0}\right)_{X}+D\left(U A_{0}\right)_{X}+D V A_{0, y}\right], \\
\mathrm{i} \Omega D B_{1}+M_{1, y} & =-\left[D U B_{0, X}+D\left(V B_{0}\right)_{y}\right], \\
\mathrm{i} \Omega\left(M_{1}-C^{2} N_{1}\right) & =0,
\end{aligned}
$$

with boundary conditions

$$
B_{1}(X, g)-g_{X} A_{0}(X, g)=0, \quad B_{1}(X, h)-h_{X} A_{0}(X, h)=0,
$$

yielding

$$
\begin{aligned}
& M_{1, y}(X, g)=-D U B_{0, X}-\mathrm{i} \mu M_{0} g_{X}, \\
& M_{1, y}(X, h)=-D U B_{0, X}-\mathrm{i} \mu M_{0} h_{X} .
\end{aligned}
$$

So $M_{0}=C^{2} N_{0}$ and $M_{1}=C^{2} N_{1}$. After combining the equations of (49) we obtain an inhomogeneous Pridmore-Brown equation for $M_{1}$

$$
\begin{aligned}
& \Omega^{2}\left(\frac{M_{1, y}}{\Omega^{2}}\right)_{y}+\left(\frac{\Omega^{2}}{C^{2}}-\mu^{2}\right) M_{1}=\mathrm{i} \Omega\left(U \frac{M_{0}}{C^{2}}\right)_{X}+\mathrm{i} \Omega\left(V \frac{M_{0}}{C^{2}}\right)_{y}+\mathrm{i} \Omega\left(D A_{0}\right)_{X} \\
& \quad+\mathrm{i} \mu D\left(\frac{M_{0}}{D}\right)_{X}+\mathrm{i} \mu D\left(U A_{0}\right)_{X}+\mathrm{i} \mu D V A_{0, y}-\Omega^{2}\left(\frac{D}{\Omega^{2}}\left[U B_{0, X}+\left(V B_{0}\right)_{y}\right]\right)_{y} .
\end{aligned}
$$

We are not aiming to solve this equation (it would involve again undetermined homogeneous solutions). However, we may derive a solvability condition on $M_{0}$ for $M_{1}$ to exist. For this we combine equations (52) and (39) as follows

$$
\mathrm{i} \int_{g}^{h} \Omega^{-2}\left(\left[\text { equation 52] } M_{0}-\left[\text { equation 39] } M_{1}\right) \mathrm{d} y,\right.\right.
$$

9 of 17 
to obtain

$$
\begin{aligned}
\left.\mathrm{i} \int_{g}^{h}\left(\frac{M_{0} M_{1, y}-M_{1} M_{0, y}}{\Omega^{2}}\right)_{y} \mathrm{~d} y=\mathrm{i} \frac{M_{0} M_{1, y}}{\Omega^{2}}\right]_{y=g}^{y=h} & \\
= & {\left[\mathrm{i} \frac{D U M_{0}}{\Omega^{2}} B_{0 y}+\frac{\mu M_{0}^{2}}{\Omega^{2}}\right]_{y=h} h_{X}-\left[\mathrm{i} \frac{D U M_{0}}{\Omega^{2}} B_{0 y}+\frac{\mu M_{0}^{2}}{\Omega^{2}}\right]_{y=g} g_{X} } \\
=\int_{g}^{h} & {\left[-\frac{M_{0}}{\Omega}\left(U \frac{M_{0}}{C^{2}}\right)_{X}-\frac{M_{0}}{\Omega}\left(D A_{0}\right)_{X}-\frac{M_{0}}{\Omega}\left(V \frac{M_{0}}{C^{2}}\right)_{y}-\frac{\mu M_{0} D}{\Omega^{2}}\left(\frac{M_{0}}{D}\right)_{X}\right.} \\
& \left.\quad-\frac{\mu M_{0} D}{\Omega^{2}}\left(U A_{0}\right)_{X}-\frac{\mu M_{0} D}{\Omega^{2}} V A_{0, y}+\mathrm{i} D \frac{M_{0 y}}{\Omega^{2}}\left(U B_{0, X}+\left(V B_{0}\right)_{y}\right)\right] \mathrm{d} y .
\end{aligned}
$$

We can further evaluate and use

$$
A_{0}=\frac{\mu M_{0}}{D \Omega}-\frac{\sigma M_{0 y}}{D \Omega^{2}}, \quad B_{0}=\mathrm{i} \frac{M_{0 y}}{D \Omega}, \quad B_{0 y}=-\mathrm{i}\left(\frac{\Omega^{2}}{C^{2}}-\mu^{2}\right) \frac{M_{0}}{D \Omega}-\mathrm{i} \frac{\mu \sigma M_{0 y}}{D \Omega^{2}},
$$

to get, eventually,

$$
\begin{aligned}
& \frac{\mathrm{d}}{\mathrm{d} X} \int_{g}^{h}\left(\mu+\frac{U \Omega}{C^{2}}\right) \frac{M_{0}^{2}}{\Omega^{2}} \mathrm{~d} y=\int_{0}^{h}\left[\left(\omega \mu \frac{D_{X}}{D}+2 \mu^{3} \sigma \frac{V}{\Omega}-\left(\frac{\Omega^{2}}{C^{2}}-\mu^{2}\right)\left(U \frac{\Omega_{X}}{\Omega}+V_{y}\right)\right) \frac{M_{0}^{2}}{\Omega^{3}}\right. \\
&+\left(\omega \sigma_{X}-2 \omega \sigma \frac{\Omega_{X}}{\Omega}+\right.\left.2 \mu \sigma U_{X}-2 \mu^{2} \sigma^{2} \frac{V}{\Omega}\right) \frac{M_{0} M_{0 y}}{\Omega^{4}}+\left(U \frac{D_{X}}{D}+U \frac{\Omega_{X}}{\Omega}-V_{y}\right) \frac{M_{0 y}^{2}}{\Omega^{3}} \\
&+\left.U\left(\frac{\Omega^{2}}{C^{2}}-\mu^{2}\right) \frac{M_{0} M_{0 X}}{\Omega^{3}}+\omega \sigma \frac{M_{0} M_{0 y X}}{\Omega^{4}}-U \frac{M_{0 y} M_{0 y X}}{\Omega^{3}}\right] \mathrm{d} y .
\end{aligned}
$$

After identifying $M_{0}=Q(X) \psi(X, y)$, and defining $\psi$ (as long as $\psi$ is differentiable, any definition will do) such that

$$
\int_{g}^{h}\left(\mu+\frac{\Omega U}{C^{2}}\right) \frac{\psi^{2}}{\Omega^{2}} \mathrm{~d} y=D,
$$

we have ${ }^{2}$

$$
\begin{aligned}
\psi(X, y) & =\frac{\eta^{\prime}(\xi)-b \xi \eta(\xi)}{\sqrt{\int_{g}^{h}\left(\mu+\frac{\Omega U}{C^{2}}\right) \frac{\left(\eta^{\prime}-b \xi \eta\right)^{2}}{D \Omega^{2}}} \mathrm{~d} y}, \\
\psi_{y}(X, y) & =\left(\frac{ \pm 2}{\mu \sigma C}\right)^{\frac{1}{2}} \mu \sigma \frac{\frac{1}{4} \xi^{2} \eta(\xi)+b \xi \eta^{\prime}(\xi)}{\sqrt{\int_{g}^{h}\left(\mu+\frac{\Omega U}{C^{2}}\right) \frac{\left(\eta^{\prime}-b \xi \eta\right)^{2}}{D \Omega^{2}}} \mathrm{~d} y} .
\end{aligned}
$$

\footnotetext{
${ }^{2}$ For a right-running hard-wall mode with $\mu>0$, the integral will be positive and the corresponding $Q$ will be real and positive, but for left-running modes or lined-wall modes $Q$ may be complex. In that case we have to make sure to remain on the same square root branch, such that $Q$ is continuous in $X$.
} 
Hence, we obtain after some re-organisation

$$
\begin{gathered}
Q_{X} D=Q \int_{g}^{h}\left[\left(2 \mu^{3} \sigma V+\left(\frac{\Omega^{2}}{C^{2}}-\mu^{2}\right)\left(U_{X} \Omega-U \Omega_{X}\right)\right) \frac{\psi^{2}}{\Omega^{4}}\right. \\
+\left(\omega \sigma_{X}-2 \omega \sigma \frac{\Omega_{X}}{\Omega}+2 \mu \sigma U_{X}-2 \mu^{2} \sigma^{2} \frac{V}{\Omega}\right) \frac{\psi \psi_{y}}{\Omega^{4}} \\
+\left(U \frac{D_{X}}{D}+U \frac{\Omega_{X}}{\Omega}-V_{y}\right) \frac{\psi_{y}^{2}}{\Omega^{3}} \\
\left.+U\left(\frac{\Omega^{2}}{C^{2}}-\mu^{2}\right) \frac{\psi \psi_{X}}{\Omega^{3}}+\omega \sigma \frac{\psi \psi_{y X}}{\Omega^{4}}-U \frac{\psi_{y} \psi_{y X}}{\Omega^{3}}\right] \mathrm{d} y \\
-Q_{X} \int_{g}^{h}\left[\omega \mu \frac{\psi^{2}}{\Omega^{3}}-\omega \sigma \frac{\psi \psi_{y}}{\Omega^{4}}+U \frac{\psi_{y}^{2}}{\Omega^{3}}\right] \mathrm{d} y,
\end{gathered}
$$

which is an equation for $Q$ of the form

$$
D Q_{X}=f_{1} Q-f_{2} Q_{X},
$$

with $f_{1}(X)$ and $f_{2}(X)$ given by the integrals in (57). Its solution is

$$
Q(X)=Q_{0} \exp \left(\int_{0}^{X} \frac{f_{1}(z)}{D(z)+f_{2}(z)} \mathrm{d} z\right),
$$

where $Q_{0}=Q(0)$ is some integration constant. This completes the leading order solution of the slowly varying modes. From $M_{0}=Q \psi$ the other amplitudes $A_{0}, B_{0}, N_{0}$ and $T_{0}$ follow.

In agreement with the related analyses of Peake and Cooper [13, 20], and [8], the resulting equation for $Q$ is not as neat as for example for the potential flow problem considered in $[11,15]$. Apparently, there is no really conserved "adiabatic invariant", if there is, like we have here, no conserved acoustic energy [24]. Only if both mean flow and perturbation field are irrotational, i.e. if $\sigma=0$ and there is a velocity potential function $\Phi$ such that $A=-\mathrm{i} \mu \Phi+\varepsilon \Phi_{X}$ and $B=\Phi_{y}$, we can rewrite (cf. [11])

$$
D\left[\Phi_{y y}+\left(\frac{\Omega^{2}}{C^{2}}-\mu^{2}\right) \Phi\right]=\frac{\mathrm{i} \varepsilon}{\Phi}\left[\frac{\partial}{\partial X}\left(\left(\mu+\frac{U \Omega}{C^{2}}\right) D \Phi^{2}\right)+\frac{\partial}{\partial y}\left(\frac{V \Omega}{C^{2}} D \Phi^{2}\right)\right],
$$

and we can derive the adiabatic invariant

$$
\int_{g}^{h}\left(\mu+\frac{U \Omega}{C^{2}}\right) D \Phi^{2} \mathrm{~d} y=\text { constant. }
$$




\section{Examples and applications}

Numerical evaluation of the above solution requires routines for the Parabolic Cylinder Functions. We used the freely available Matlab-files by Bandres et al. [25, 26]. They work well with high accuracy for real values of the variables. There are, however, inherent limitations in the present application, due to the transformation from $y$ to $\xi$, involving a division by $\sigma$, and the highly oscillatory behaviour $\left(\sim \cos \frac{1}{4} \xi^{2}\right)$ of the functions. It makes the routines, without further extensions based on asymptotic expansions or otherwise, only applicable to moderate problem parameter values.

Both the numerical integrations in $y$ and $x$, and the $x$-derivatives of the modal shape functions $\psi$ and $\psi_{y}$ were done numerically by trapezoidal rule and finite differences, based on a uniform grid of 600 steps in $x$-direction and a non-uniform (duct-following) grid of 100 steps in $y$-direction.

A typical and relevant example is pictured below. The mean flow is described by the problem parameters

$$
\lambda=0.5, \quad \gamma=1.4, \quad D_{\text {in }}=1, \quad \tau_{\text {in }}=0.2, \quad \mathcal{F}=0.4496, \quad E=2.52,
$$

and the geometry by

$$
h(x)=1-\frac{1}{8}(1+\tanh (x), \quad g(x)=0, \quad-3<x<3 .
$$

For the acoustic part we considered right-running modes with $\omega=13$ and left-running modes with $\omega=2$ and $\omega=4$. (The initial value $Q_{0}$ of the amplitude was taken unity, but this plays no role in a linear problem.) We have four cut-on right-running modes with $\omega=13$ and considered ${ }^{3}$ one cut-on left-running mode for $\omega=2$ and $\omega=4$ each.

We see that the effects of the sheared mean flow properties are various. Especially for the lower modal orders the asymmetry of the flow is reflected in asymmetric mode shapes. Sometimes, $\omega=13$ (1) and $\omega=2$, a kind of tunneling effect is visible, in the sense that the mode is only observable along one side of the duct and is negligible along the other.

Mode $\omega=13$ (4) changes from well cut-on to almost cut-off, showing a clear increase of the modal wave length. Indeed, in general the right-running modal wave numbers $\mu$ decrease with a narrower duct. This effect is not seen with the left-running wave numbers. Apparently, the effect of the narrowing duct is compensated by the increase of the mean flow speed.

As far as the right-running modes are concerned, in all cases the normalisation integral (55) is positive for all $x$. Of the left-running modes, the normalisation integral (55) is, for all $x$, positive for $\omega=2$ and negative for $\omega=4$.

\section{Conclusions}

The understanding of sound propagation in ducts is greatly enhanced if we can model the duct straight (in $x$ direction, say), with a uniform medium and uniform boundary conditions, and a constant cross section. In that case self-similar solutions exist of the form $\psi(x-V t, y, z)$, which reduce in case of harmonic time dependence to what is commonly known as duct modes, of the form $\mathrm{e}^{\mathrm{i} \omega t-\mathrm{i} k x} \hat{\psi}(y, z ; k)[2]$. The modal shape functions $\hat{\psi}$ are eigensolutions of the Laplace

\footnotetext{
${ }^{3}$ Due to numerical limitations of the Parabolic Cylinder Function routines, it was easier to evaluate examples of right-running modes than left-running modes. With $\mu>0$ the variable $\xi \sim \omega-\mu(\tau+\sigma)$ is not as big as with $\mu<0$.
} 

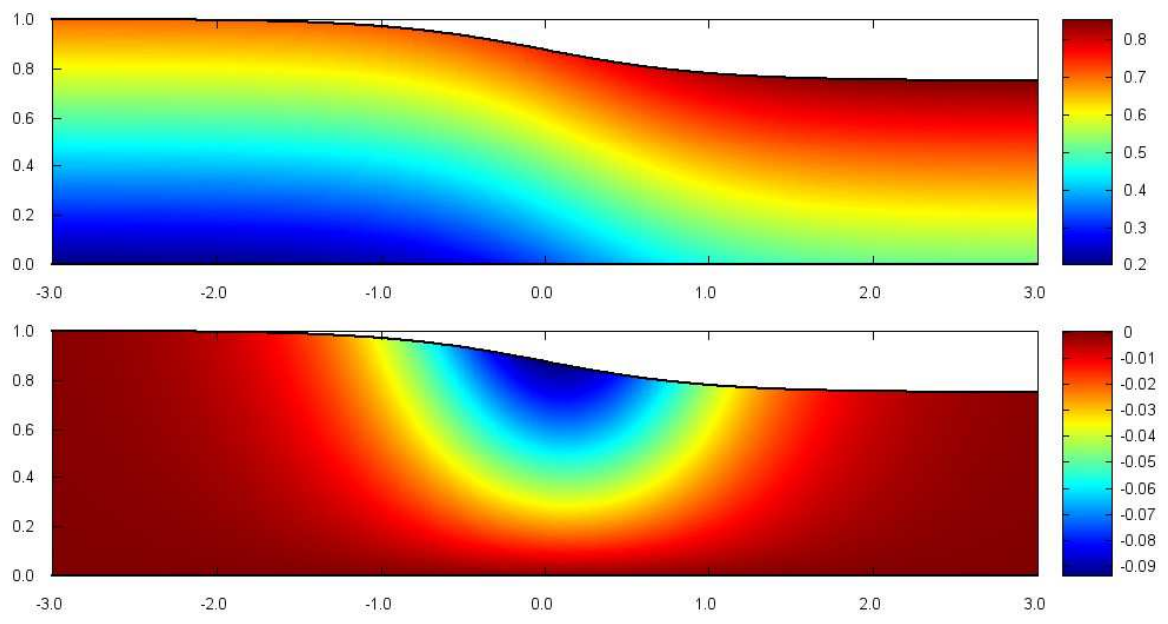

Figure 2. Mean flow $U$ and $V$ in $x, y$-domain

operator $-\nabla^{2}$ in $y$ and $z$, with an eigenvalue related to wave number $k$. The possible modes are infinite in number but the wave number spectrum is discrete. In fact, the modes form a complete basis to construct any possible solution by linear combination.

For simple duct cross sections (cylindrical, rectangular, 2D) and simple enough boundary conditions, these eigensolutions can be determined analytically, possibly with an algebraic equation to be solved numerically.

This scenario remains valid in practically unchanged form for flow ducts with a uniform mean flow [3]. For flow ducts with non-uniform but parallel flow we still have self-similar solutions of modal form, but the prevailing equation, called Pridmore-Brown equation (in one or other form a generalisation of the Helmholtz equation), is more complicated and in general only solvable numerically [9]. Also is the wave number spectrum not necessarily discrete and the modal basis not complete [7]. Some solutions cannot be written as a modal sum. Nevertheless, the PridmoreBrown modes are of primary importance to describe sound propagation in ducts with sheared mean flow. Therefore any analytic solution of the Pridmore-Brown equation is of interest, since they are rare. For example, as was found by Goldstein \& Rice [21], for linear sheared mean flow (linear in $y$, uniform in $z$ ) the Pridmore-Brown equation can be reduced to a form of the equation for Parabolic Cylinder Functions [23]. Although not as common as (for example) Bessel functions, these functions are standard functions and part of, or available through, many mathematical software packages.

A variation on the theme of modal propagation of sound in ducts is the idea of slowly varying modes (that is, a WKB approximation of a mode-like sound wave) in a slowly varying duct. Without mean flow this is relatively straightforward because the main equation remains the Helmholtz equation, and the solution [10] has been available already since 1973. With mean flow, the problem is more complicated because also the mean flow is varying with the varying duct. In particular, the slowly varying solution of the mean flow equations has to be available in some form to make a consistent WKB analysis possible.

For potential (irrotational) flow, the slowly varying mean flow is nearly uniform and already known as the solution of the (quasi) 1D gas dynamics equations [11, 15]. For sheared (rotational) flow, it appears to be far more difficult to obtain solutions of slowly varying type. Cooper and 

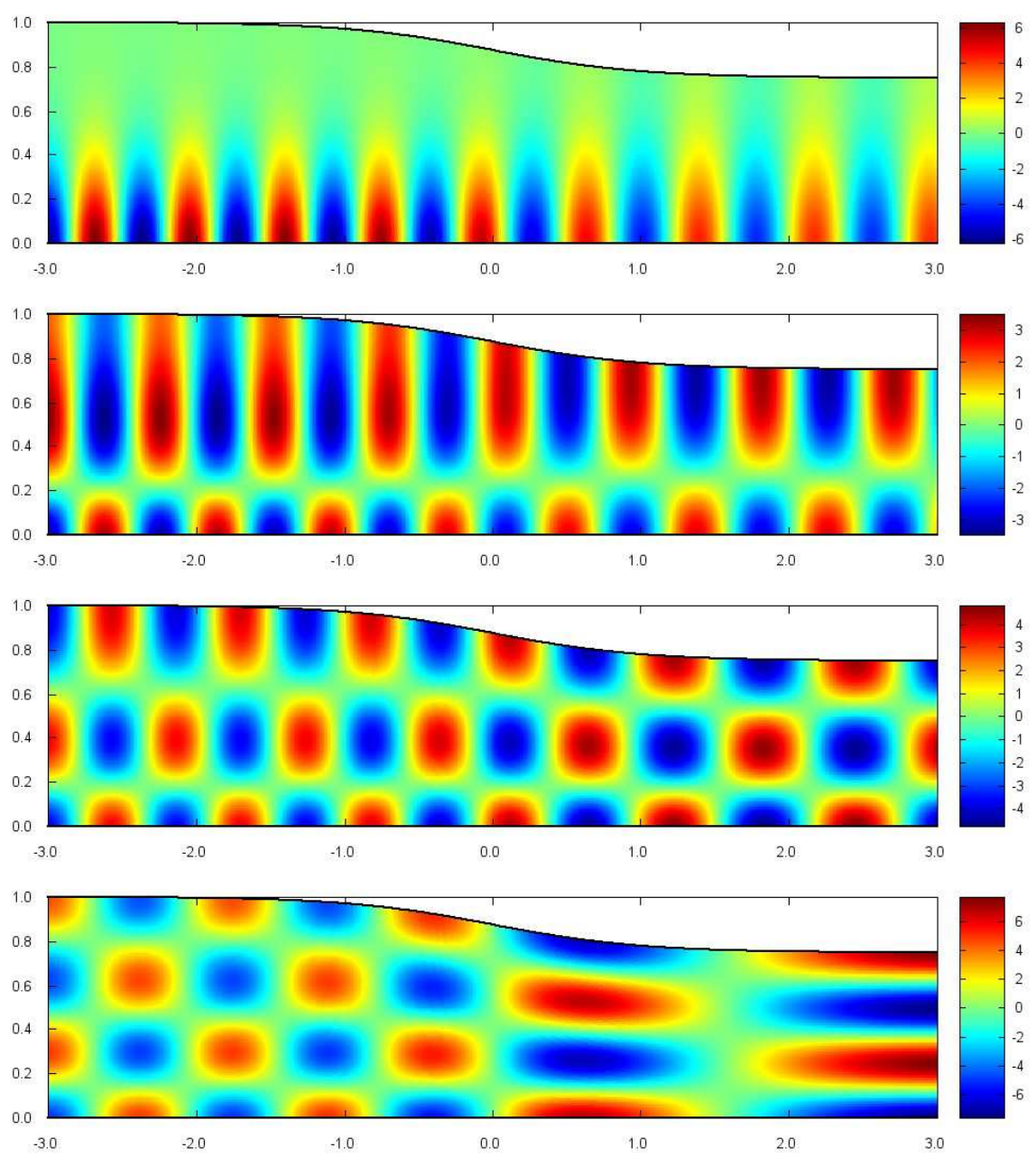

Figure 3. Right-running modal pressure fields for $\omega=13$ and increasing radial mode number
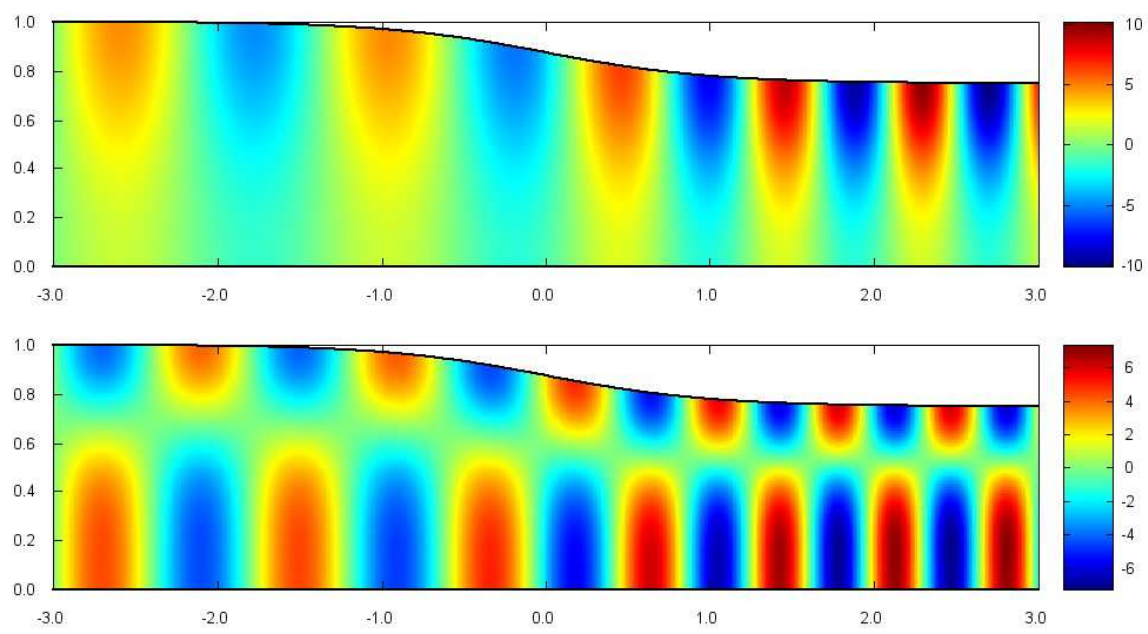

Figure 4. Left-running modal pressure fields for $\omega=2$ (t) and $\omega=4$ (b) 


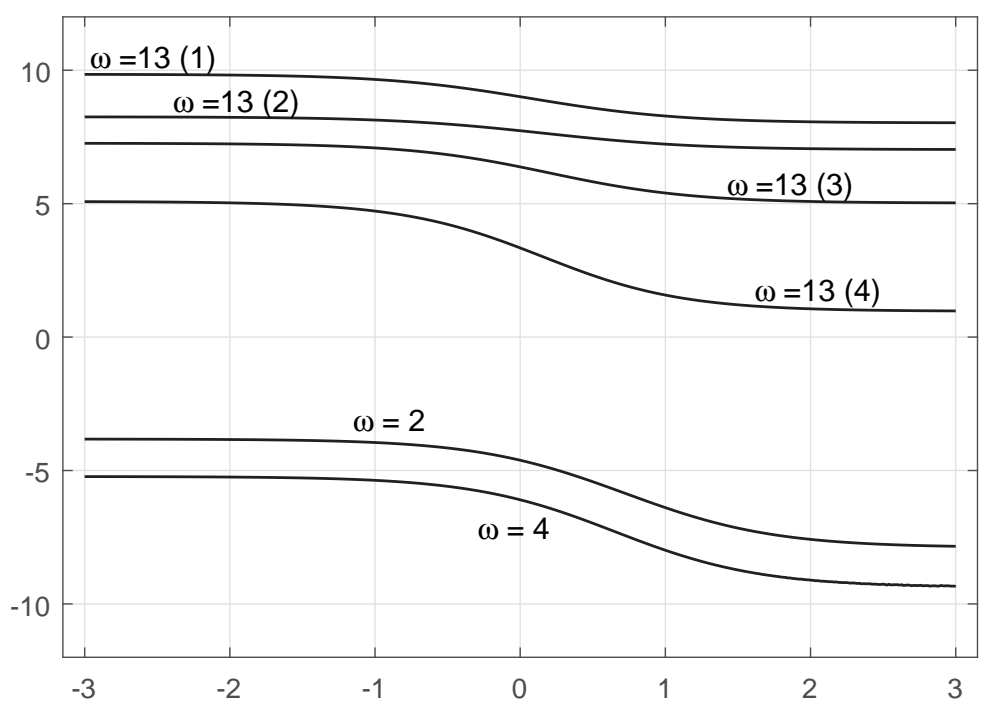

Figure 5. Axial wave numbers $\mu$ as function of $x$
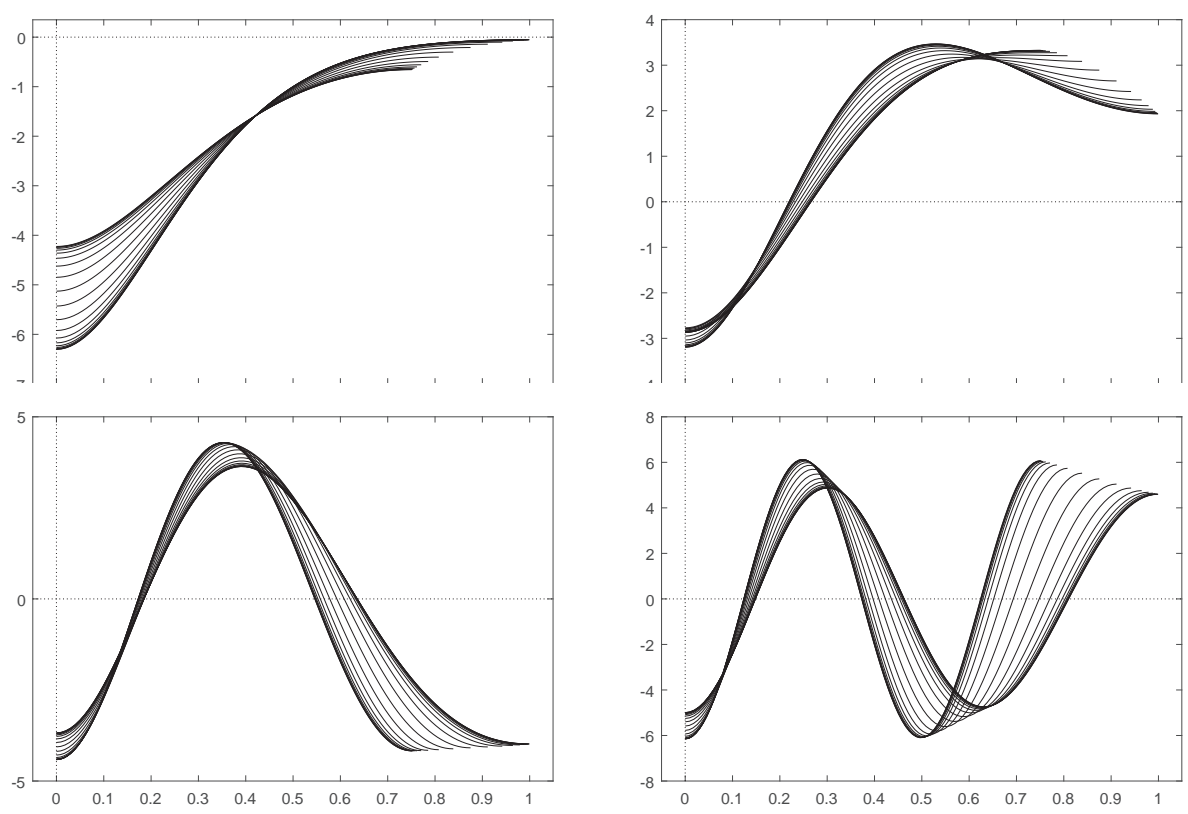

Figure 6. Right-running modal functions $\psi(y, x)$ for $\omega=13$, as function of $y-g$ for varying $x$
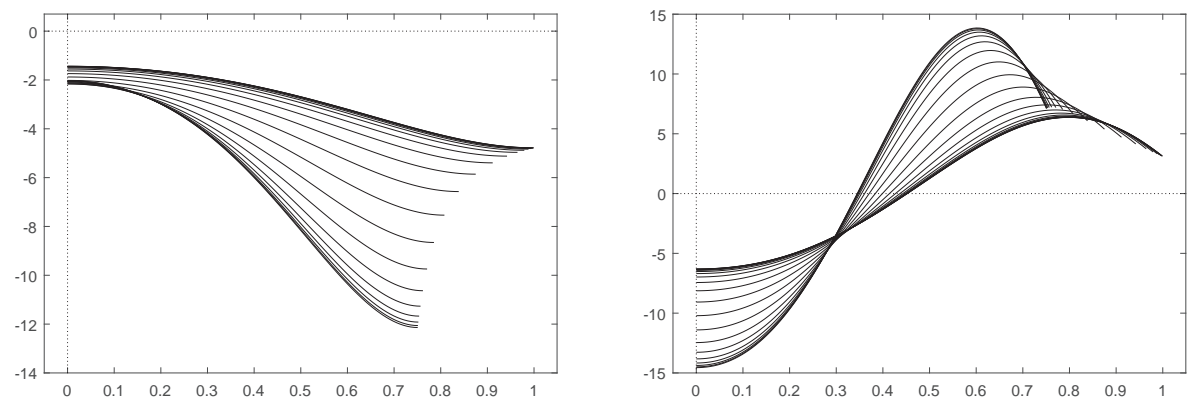

Figure 7. Left-running modal functions $\psi(y, x)$ for $\omega=2$ (l) and $\omega=4(\mathbf{r})$, as function of $y-g$ for varying $x$

\section{5 of 17}

American Institute of Aeronautics and Astronautics Paper AIAA-2016-2925 
Peake [20] presented slowly varying modes with a mean flow model that includes swirl, in slowly varying circular ducts. The steady Euler equations were reduced to a system of (nonlinear) ordinary differential equations for the stream function, and were subsequently worked out for a uniform axial velocity with rigid-body rotation type of swirl.

However, as we show here, there is also a slowly varying mean flow possible for a flow of linear shear $U=\tau+\sigma y$, where only the base $\tau$ and shear coefficient $\sigma$ vary with the geometry. Otherwise, the axial velocity remains linear in $y$. This property usefully fits with the available Parabolic Cylinder-type of solution of the Pridmore-Brown equation.

In this paper we aimed to extend the number of possible models allowing analytic solutions, by combining the slowly varying mean shear flow with the WKB solution of the Pridmore-Brown equation based on the Parabolic Cylinder functions. The results are satisfying but as yet only for not too extreme parameter values, because the available routines, although very accurate, are used in a way that often a numerically higher accuracy (preferably obtained by asymptotic expansions) is required.

\section{References}

1 S.W. Rienstra, 2003, A Classification of Duct Modes Based on Surface Waves, Wave Motion, 37, 119-135.

2 S.W. RiEnstra, 2015, Fundamentals of Duct Acoustics, Von Karman Institute Lecture Notes.

3 S.W. RIENSTRA and A. HIRsChBERG, 1992, An Introduction to Acoustics, TR-IWDE-92-06, Technische Universiteit Eindhoven, 1992-2004-2015, http://www.win.tue.nl/ sjoerdr/papers/boek.pdf

4 D. Pridmore-Brown, 1958, Sound Propagation in a Fluid Flowing Through an Attenuating Duct, Journal of Fluid Mechanics, 4 (4), 393-406.

5 G.G. Vilenski and S.W. RiEnstra, 2007, Numerical Study of Acoustic Modes in Ducted Shear Flow, Journal of Sound and Vibration, 307 (3-5), 610-626.

6 G.G. VilensKI and S.W. RIENSTRA, 2007, On Hydrodynamic and Acoustic Modes in a Ducted Shear Flow with Wall Lining, Journal of Fluid Mechanics, 583, 45-70.

7 E.J. BRAmbley, S.W. Rienstra and M. DARAU, 2012, The critical layer in linear-shear boundary layers over acoustic linings, Journal of Fluid Mechanics, 710, 545-568.

8 M. Oppeneer, S.W. Rienstra, W. LaZeroms, R.M.M. Mattheij and P. SiJtsma, 2011, Acoustic modes in a duct with slowly varying impedance and non-uniform mean flow and temperature, paper AIAA 2011-2871 of the 17th AIAA/CEAS Aeroacoustics Conference, 6-8 June 2011, Portland, Oregon, USA

9 M. Oppeneer, S.W. Rienstra and P. Sijtsma, 2016, Efficient Mode-Matching Based on Closed Form Integrals of Pridmore-Brown Modes, AIAA Journal, 54 (1), 266-279.

10 A.H. NAYFEH and D.P. TELIONIS, 1973, Acoustic propagation in ducts with varying cross sections, Journal of the Acoustical Society of America, 54 (6), 1654-1661.

11 S.W. Rienstra, 1999, Sound Transmission in Slowly Varying Circular and Annular Ducts with Flow, Journal of Fluid Mechanics, 380, 279-296.

12 S.W. Rienstra, 2000, Cut-on, Cut-off Transition of Sound in Slowly Varying Flow Ducts, contribution to the David Crighton memorial issue of Aerotecnica Missili E Spazio (Journal of the Associazione Italiana di Aeronautica e Astronautica AIDAA), (ed. L. Morino and N. Peake), 79 (3-4), July-December 2000, 93-96.

13 N. Peake and A.J. Cooper, 2001, Acoustic Propagation In Ducts With Slowly Varying Elliptic Cross-Section, Journal of Sound and Vibration, 243 (3), 381-401.

14 N.C. OvEnDEN, 2002, Near cut-on/cut-off transitions in lined ducts with flow, paper AIAA 2002-2445 of the 8th AIAA/CEAS Aeroacoustics Conference in Breckenridge, CO, 17-19 June 2002.

15 S.W. Rienstra, 2003, Sound Propagation In Slowly Varying Lined Flow Ducts Of Arbitrary Cross Section, Journal of Fluid Mechanics, 495, 157-173.

16 N.C. Ovenden, W. Eversman, and S.W. Rienstra, 2004, Cut-on cut-off transition in flow ducts: Comparing multiple-scales and finite-element solutions. paper AIAA 2004-2945 of the 10th AIAA/CEAS Aeroacoustics Conference, Manchester, UK, May 10-12 2004. 
17 N.C. Ovenden, and S.W. Rienstra, 2004, Mode-matching strategies in slowly varying engine ducts. AIAA Journal, 42 (9), 1832-1840.

18 S.W. RiEnstra and W. Eversman, 2001, A Numerical Comparison Between Multiple-Scales and FEM Solution for Sound Propagation in Lined Flow Ducts, Journal of Fluid Mechanics, 437, 367-384.

19 N.C. OvEnDEN, 2005 A uniformly valid multiple scales solution for cut-on cut-off transition of sound in flow ducts. Journal of Sound and Vibration, 286, 403-416.

20 A.J. Cooper and N. Peake, 2001, Propagation of Unsteady Disturbances in a Slowly Varying Duct with Mean Swirling Flow, Journal of Fluid Mechanics, 445, 207-234.

21 M. Goldstein and E. RICE, 1973, Effect of Shear on Duct Wall Impedance, Journal of Sound and Vibration, 30 (1), 79-84.

22 M.K. MYers, 1980, On the Acoustic Boundary Condition in the Presence of Flow. Journal of Sound and Vibration, 71 (3), 429-434.

23 M. AbRamowitz and I.A. STEgUn, 1964, Handbook of Mathematical Functions, National Bureau of Standards, Dover Publications, Inc., New York.

24 M.K. MYERS, 1991, Transport of energy by disturbances in arbitrary steady flows, Journal of Fluid Mechanics, 226, 383-400.

25 M.A. Bandres, J.C. GutiérREZ-VegA, and S. ChÁVEZ-Cerda, Parabolic nondiffracting optical wavefields, Optics Letters, 29 (1), 44-46.

26 M.A. BANDres and B.M. RodríGUEZ-LARA, 2013 Nondiffracting accelerating waves: Weber waves and parabolic momentum, New Journal of Physics, 15 (013054). 
PREVIOUS PUBLICATIONS IN THIS SERIES:

\begin{tabular}{|c|c|c|c|}
\hline Number & Author(s) & Title & Month \\
\hline 16-09 & $\begin{array}{l}\text { B. Bagheri } \\
\text { M.E.J. Karttunen } \\
\text { B. Baumeier }\end{array}$ & $\begin{array}{l}\text { Solvent effects on optical } \\
\text { excitations of poly para } \\
\text { phenylene ethynylene } \\
\text { studied by QM/MM } \\
\text { simulations based on Many- } \\
\text { Body Green's Functions } \\
\text { Theory }\end{array}$ & $\overline{\text { May '16 }}$ \\
\hline $16-10$ & $\begin{array}{l}\text { B. Bagheri } \\
\text { M.E.J. Karttunen } \\
\text { B. Baumeier }\end{array}$ & $\begin{array}{l}\text { Getting excited: Challenges } \\
\text { in quantum-classical studies } \\
\text { of excitons in polymeric } \\
\text { systems }\end{array}$ & May '16 \\
\hline $16-11$ & $\begin{array}{l}\text { B. Plestenjak } \\
\text { M.E. Hochstenbach }\end{array}$ & $\begin{array}{l}\text { Roots of bivariate } \\
\text { polynomial systems } \\
\text { via determinantal } \\
\text { representations }\end{array}$ & May ' 16 \\
\hline $16-12$ & $\begin{array}{l}\text { P.G.Th. van der Varst } \\
\text { A.A.F. van de Ven } \\
\text { G. de With }\end{array}$ & $\begin{array}{l}\text { Load-depth sensing of } \\
\text { isotropic, linear viscoelastic } \\
\text { materials using rigid } \\
\text { axisymmetric indenters }\end{array}$ & May '16 \\
\hline $16-13$ & S.W. Rienstra & $\begin{array}{l}\text { Sound Propagation in Slowly } \\
\text { Varying 2D Duct with Shear } \\
\text { Flow }\end{array}$ & May '16 \\
\hline
\end{tabular}

